\title{
Absence of vaccine-enhanced RSV disease and changes in pulmonary dendritic cells with adenovirus-based RSV vaccine
}

\author{
Anja Krause', Yaqin $\mathrm{Xu}^{2}$, Sara Ross ${ }^{2}$, Wendy $\mathrm{Wu}^{2}, \mathrm{Ju}_{\mathrm{J}} \mathrm{Jh}^{1}$ and Stefan Worgall ${ }^{1,2^{*}}$
}

\begin{abstract}
The development of a vaccine against respiratory syncytial virus (RSV) has been hampered by the risk for vaccineenhanced RSV pulmonary disease induced by immunization with formalin-inactivated RSV (FIRSV). This study focuses on the evaluation of vaccine-enhanced pulmonary disease following immunization with AdF.RGD, an integrin-targeted adenovirus vector that expresses the RSV F protein and includes an RGD (Arg-Gly-Asp) motif. Immunization of BALB/c mice with AdF.RGD, resulted in anti-RSV protective immunity and induced increased RSVspecific IFN- $\gamma$ T cell responses compared to FIRSV. RSV infection 5 wk after immunization with FIRSV induced pulmonary inflammatory responses in the lung, that was not observed with AdF.RGD. Additionally, In the FIRSVimmunized mice following infection with RSV, pulmonary DC increased and Tregs decreased. This suggests that distinct responses of pulmonary DC and Tregs are a features of vaccine-enhanced RSV disease and that immunization with an RGD-modified Ad vaccine does not trigger vaccine-enhanced disease.
\end{abstract}

\section{Introduction}

RSV is a leading cause of severe viral respiratory disease in infants and children [1]. A major obstacle in the development of an RSV vaccine has been vaccineenhanced disease triggered by immunization with inactivated virus antigen [2,3]. This aberrant immunemediated response is characterized by infiltration of neutrophils and eosinophils, increased complement-fixing antibody titers and lymphoproliferative responses [4-7]. The exact mechanism has not been fully elucidated. An altered pattern of CD4 lymphocyte activation with eosinophil recruitment and Th2-type-predominant cytokine production (IL-4, IL-5, IL-13 and eotaxin) suggests an aberrant immune-mediated response skewed towards Th2 responses [4,8-10]. This is supported by data demonstrating that the disease following RSV infection can be transferred by RSV G protein-specific CD4 $\mathrm{T}$ cells and also occurs in the absence of CD8 T cells or IFN- $\gamma$ [11-13]. RSV-specific CD8 T cells can even inhibit the disease $[7,13,14]$. Initially, the RSV G protein itself was implicated based on studies using vaccinia virus

\footnotetext{
* Correspondence: stw2006@med.cornell.edu

'Department of Genetic Medicine, Weill Medical College of Cornell

University, New York, New York, USA

Full list of author information is available at the end of the article
}

expressing the membrane-anchored part or the entire G protein for immunization [15-17]. Recently, it has been suggested that the pathway of antigen processing, rather than the antigenic content, is responsible [4,7]. In addition, low avidity anti-RSV antibodies, which may have resulted from poor activation of toll-like receptors, have been observed in mice following immunization with FIRSV [18], the most commonly used model to study vaccine-enhanced RSV disease [4,19-21].

The RSV F protein, one of the main capsid proteins that confers protective immunity against RSV, has been a major target in RSV vaccine development [22]. Vaccination with the $\mathrm{F}$ protein generates helper $\mathrm{T}$ cell responses that are Th1 in character [23]. Adenovirus (Ad)-based gene delivery systems are promising platforms for genetic vaccines due to their ability to act as immune system adjuvants and to induce strong cellular and humoral responses against the virus and the transgene $[24,25]$ and have been used as experimental vaccines against RSV [26-32]. In part, the effectiveness of Ad-based vaccines results from the ability of Ad vectors to transfer genes to antigen presenting cells in vivo, particularly dendritic cells (DC) [33-37]. The immune response can be further enhanced to a more Th1-dominant response by modification of Ad capsid proteins to 
infect DC more efficiently [38-40]. The primary interaction of Ad with cells in vitro is through the knob domain of fiber to the coxsackievirus and adenovirus receptor (CAR) on the target cell $[41,42]$. A secondary interaction occurs between the RGD (Asp-Arg-Gly) motif in the penton base and integrins. The addition of an RGD motif (in addition to that found in the penton base) enhances infection of DC, which express low levels of CAR and high levels of surface integrins [38-40].

Virus-specific humoral and cellular adaptive immune responses are responsible for protection and recovery from RSV infection. Lung DC, as part of the pulmonary innate immune system, recognize the infection, evoke anti-viral responses and modulate the Th1/Th2 balance [43-47]. There are two major subsets of DC in the mouse, (1) the CD $11 b^{+}, C D 11 c^{\text {high }}$ conventional DC (cDC), and (2) the $\mathrm{CD}_{11 b^{\text {low- }}}, \mathrm{CD} 11 \mathrm{c}^{\text {high }}, \mathrm{B} 220^{+}$plasmacytoid DC (pDC) [48-52]. Resting lung $\mathrm{cDC}$ have been implicated in initiating a pro-allergic Th2 response in the lung and in requiring special cytokine signals to induce Th1 response, whereas $\mathrm{pDC}$ seem to have a primary role in producing IFN- $\alpha$ in response to viral infections and in promoting a Th1 response by blocking the pulmonary immune environment against a Th2 response [43,50,53-56]. PDC balance the $\mathrm{T}$ helper cell responses through cross-talk with regulatory $\mathrm{T}$ cells (Tregs). The number of $\mathrm{pDC}$ and $\mathrm{cDC}$ is increased in lung and draining lymph nodes following experimental RSV infection in mice [55,57-59] as well as in nasal washings of RSVinfected infants [60]. RSV-stimulated $\mathrm{CDC}$ seem to have immunostimulatory effects on both Th1 and Th2 responses [57]. RSV-stimulated pDC have direct antiviral activity through the release of IFN- $\alpha$ (2). Depletion of pulmonary pDC leads to an exaggerated Th2 response to RSV [55,56], whereas increasing the number of pDC by recombinant Flt3 ligand leads to an increased Th1 response [50]. Increasing $\mathrm{CDC}$ while depleting $\mathrm{pDC}$ leads to enhanced Th2-type pathology [50]. We hypothesized that the lung DC subsets stimulated in response to RSV infection in immunized animals would depend upon the T-helper cell type induced by immunization.

The present study demonstrates that immunization with the capsid-modified Ad vector AdF.RGD compared to immunization with FIRSV, at doses inducing comparable levels of anti-RSV neutralizing and protective immunity, leads to a more pronounced RSV-specific Th1 response and does not prime for vaccine-induced enhanced RSV disease. Furthermore, immunization with AdF.RGD or FIRSV results in a distinct response of pulmonary DC and Tregs that may be useful for the characterization of vaccine-enhanced pulmonary RSV disease.

\section{Results}

Immunization with AdF.RGD Leads to Enhanced Th1-Type anti-RSV Immunity and Improved Protection against RSV

We first evaluated if immunization with AdF.RGD could further polarize the anti-F immune response towards Th1 and improve the protection against RSV in comparison to immunization with the capsid-unmodified vector AdF. BALB/c mice were immunized intramuscularly with either AdF, AdF.RGD or AdNull and IL-4 responses in CD4 $\mathrm{T}$ cells and IFN- $\gamma$ responses in CD8 $\mathrm{T}$ cells against the recombinant RSV F protein (smt3RSV F) and the $\mathrm{H}-2^{\mathrm{d}}$-restricted F epitope (F85-93, KYKNAVTEL), respectively, were evaluated in lymphocytes isolated from spleen 10 days following immunization (Figure 1A,B). F protein-specific IL-4 in CD4 T cells were induced both by AdF and AdF.RGD ( $\mathrm{p}<$ 0.001 and $\mathrm{p}<0.03$ compared to AdNull, respectively; Figure 1A). However, the AdRGD-induced response was lower compared to AdF-induced response ( $p<0.002)$. In contrast, the RSV-F epitope-specific IFN- $\gamma$ response in CD8 $\mathrm{T}$ cells was increased in the mice immunized with AdF.RGD compared to mice immunized with AdF $(\mathrm{p}<0.001$; Figure 1B). This suggested that the RGD modification of the AdF vector increased the anti-RSV Th1 response and decreased the Th2 response. To evaluate if the immunization induces sufficient immunity to provide protection against a subsequent pulmonary challenge with RSV, BALB/c mice were immunized with AdF, AdF.RGD, or AdNull and challenged 5 wk later with RSV intranasally. Immunization with both AdF and AdF.RGD resulted in reduced RSV titers in the lung compared to the AdNull group ( $\mathrm{p}<0.05$, both comparisons; Figure $1 \mathrm{C}$ ). Interestingly, immunization with AdF.RGD resulted in further reduction in the RSV lung titers than immunization with $\operatorname{AdF}(\mathrm{p}<0.01)$. This suggests that the RGD modification results in increased protective immunity against RSV. Based on these results, the AdF.RGD vector was used for the following experiments.

\section{Immunization with AdF.RGD Leads to Improved Humoral anti-RSV Immunity Without Inducing Vaccine-enhanced Disease}

Humoral immune responses were evaluated following immunization with AdF.RGD in direct comparison to immunization with RSV and FIRSV, a vaccine that triggers vaccine-enhanced RSV disease. BALB/c mice immunized with AdF.RGD, RSV or FIRSV all had increased neutralizing anti-RSV titers in the serum after 4 wk compared to mice that had received the control AdNull vector $(\mathrm{p}<0.001$; Figure 2). AdF.RGD induced neutralizing anti-RSV antibodies at higher levels compared to live RSV and FIRSV-immunized mice $(\mathrm{p}<0.05)$. There was 


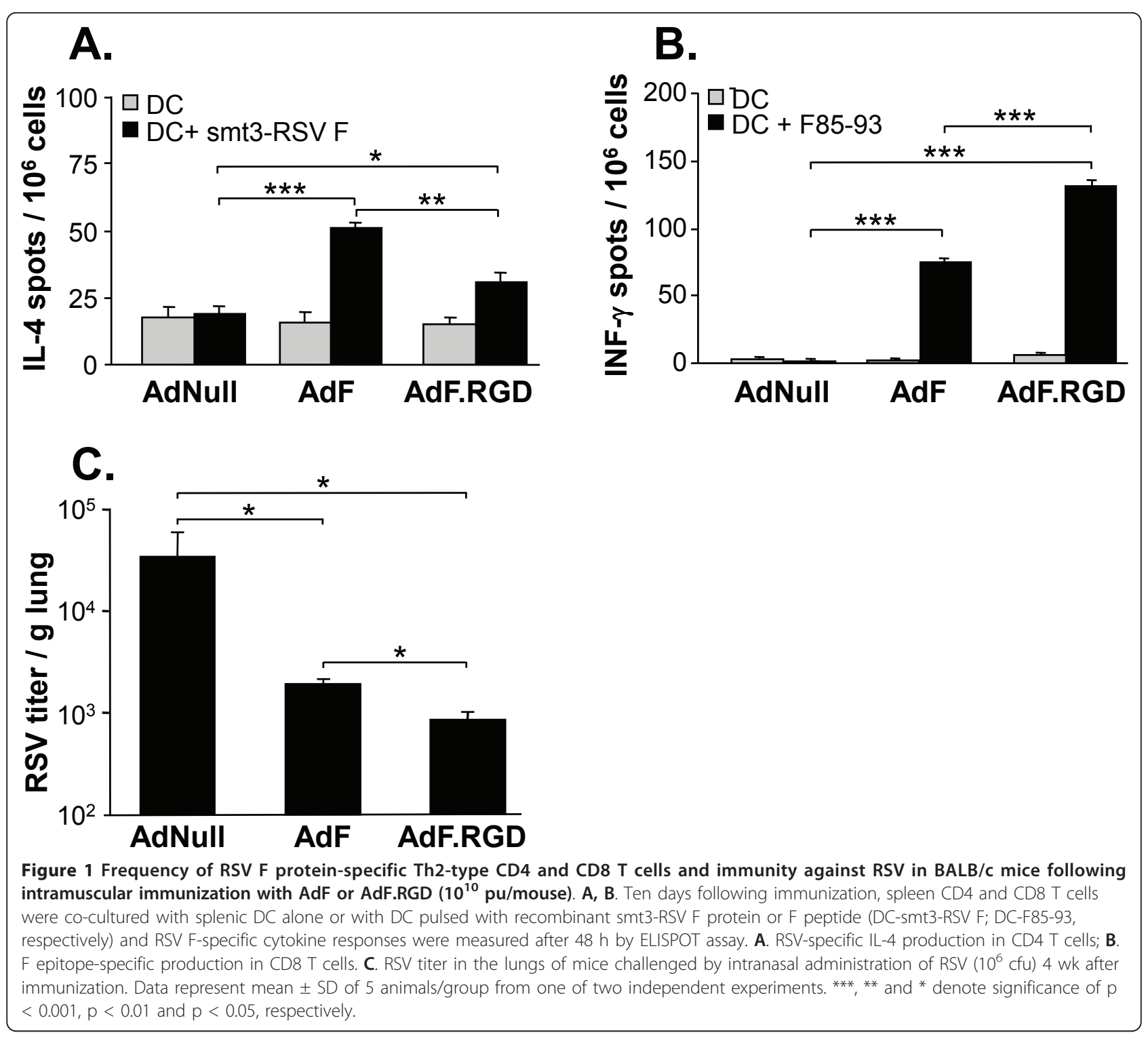

no difference in neutralizing anti-RSV titer between RSV and FIRSV-immunized animals ( $\mathrm{p}>0.1$ ).

To evaluate if immunization with AdF.RGD induces vaccine-enhanced RSV lung disease, mice were immunized with AdF.RGD, AdNull, RSV or FIRSV and then challenged with RSV at 5 wk post-immunization. Vaccine-enhanced RSV disease was assessed 6 days following RSV infection by: (1) lung histology and (2) the cellular composition of the bronchioalveolar lavage (BAL), (Figure 3), and (3) the Th2-type cytokines eotaxin, IL-4, IL10 and IL-13 in lung homogenate (Figure 4). Pronounced inflammation, consistent with vaccine-enhanced disease, was seen in the lungs of animals that had been immunized with FIRSV and had been challenged with RSV (Figure 3B). Vaccination with RSV induced moderate inflammation in the lung after RSV challenge with less infiltration of inflammatory cells compared to the FIRSV immunized animals after RSV challenge (Figure 3A). In contrast, only minimal inflammatory changes were observed in the mice immunized with AdF.RGD (Figure 3C). Analysis of the cell differential in the BAL showed an increase in eosinophils, lymphocytes and neutrophils FIRSV-immunized animals and an increase in lymphocytes in the RSV-immunized animals that had been challenged with RSV compared to those that had received AdF.RGD or AdNull (Figure 3D). Only a mild increase in the number of lymphocytes and neutrophils was observed in AdF.RGD and AdNullimmunized animals challenged with RSV compared to unchallenged animals (Figure 3D). Consistent to these findings, the levels of inflammatory cytokines like IL-4, IL-10, Il-13 and eotaxin in lung homogenate were 


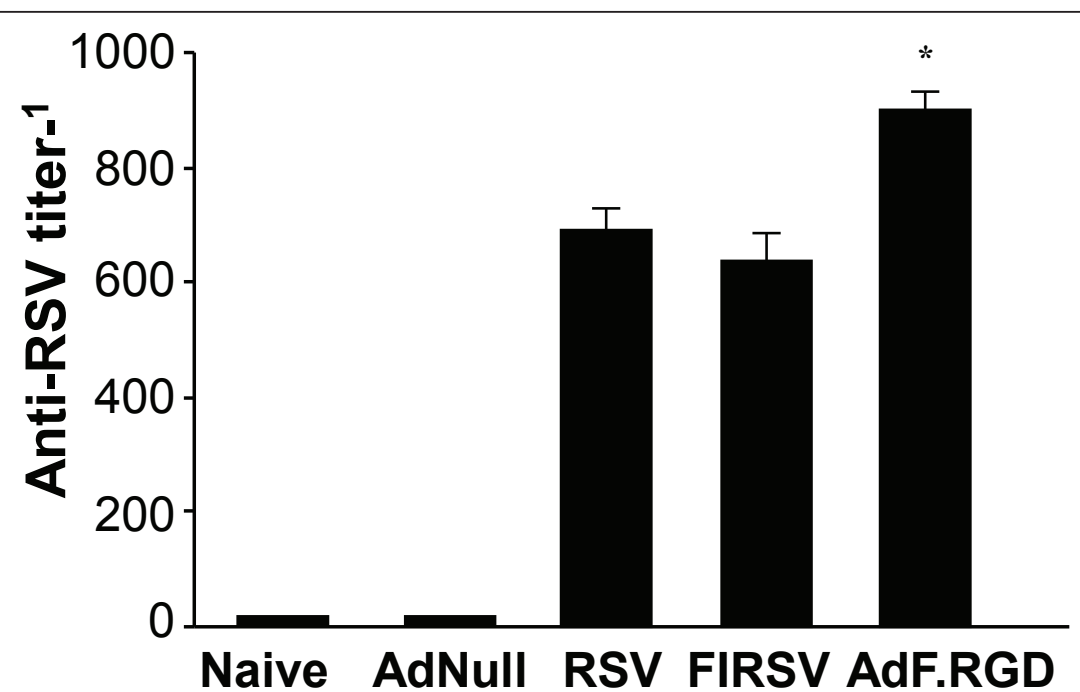

Figure 2 Protective immunity induced by AdF.RGD. BALB/c mice were immunized intramuscularly with AdNull, AdF.RGD (both at $10^{10}$ pu), formalin-inactivated RSV (FIRSV, $10^{5} \mathrm{pfu}$ ) or intranasally with RSV $\left(10^{6} \mathrm{pfu}\right.$ ) and neutralizing serum anti-RSV Titer was measured 4 wk following immunization. Data represent mean \pm SD of 5 animals/group from one of two independent experiments. ${ }^{*}$ denotes significance $p<0.05$.

increased in mice that had been immunized with FIRSV prior to RSV challenge compared to Naive, AdNull and AdF.RGD lung homogenates (eotaxin: $\mathrm{p}<0.001$, Figure 4A; IL-4: $\mathrm{p}<0.05$, Figure 4B; IL-13 and IL-10: $\mathrm{p}<$ 0.001 , Figure $4 \mathrm{C}$ and $4 \mathrm{D}$, respectively). Immunization with RSV prior to RSV challenge induced similar elevated eotaxin and IL-13 levels in the lung as seen in the FIRSV-immunized animals (Figure $4 \mathrm{~A}$ and $4 \mathrm{C}$, respectively), whereas IL-4 and IL-10 levels were not increased compared to naive mice or mice that had received
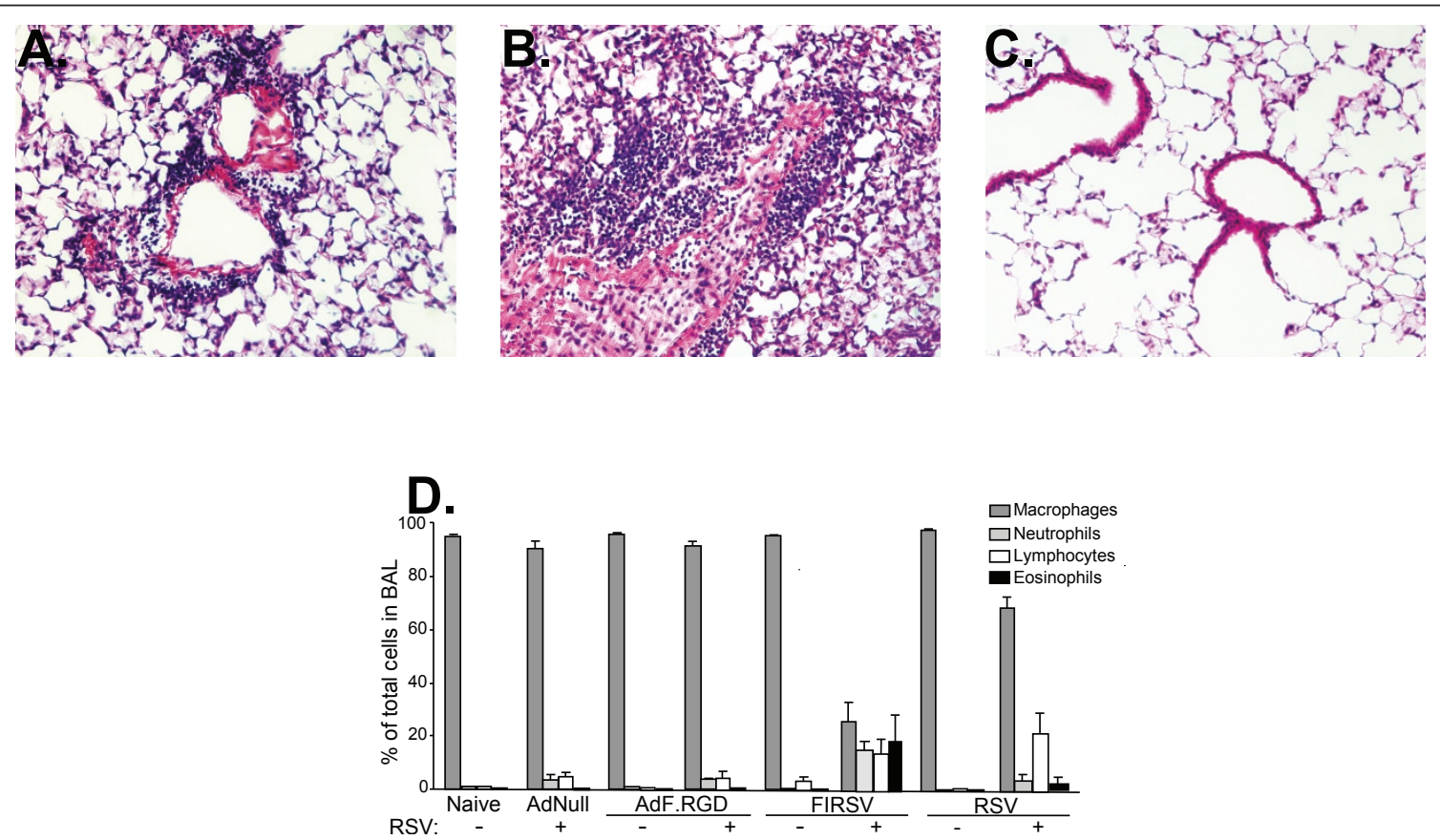

Figure 3 Absence of RSV-induced inflammatory responses in lung and BAL following immunization with AdF.RGD. BALB/C mice were immunized subcutaneously with AdNull, AdF.RGD (both at $10^{10} \mathrm{pu}$ ), formalin-inactivated RSV (FIRSV, $10^{5} \mathrm{pfu}$ ) or intranasally with RSV (10 ${ }^{6}$ pfu). Five wk later the mice were challenged intranasally with RSV ( $\left.10^{6} \mathrm{pfu}\right)$ and lungs were harvested after 6 days. A-C. Lung histology (H+E stain): A. RSV, B. FIRSV, C. AdF.RGD. D. Quantification of cells in BAL. Data for $\mathbf{D}$ are shown as mean \pm SEM of duplicate measurements of $\mathrm{n}=4$ mice/ group and represent one of two independent experiments. 


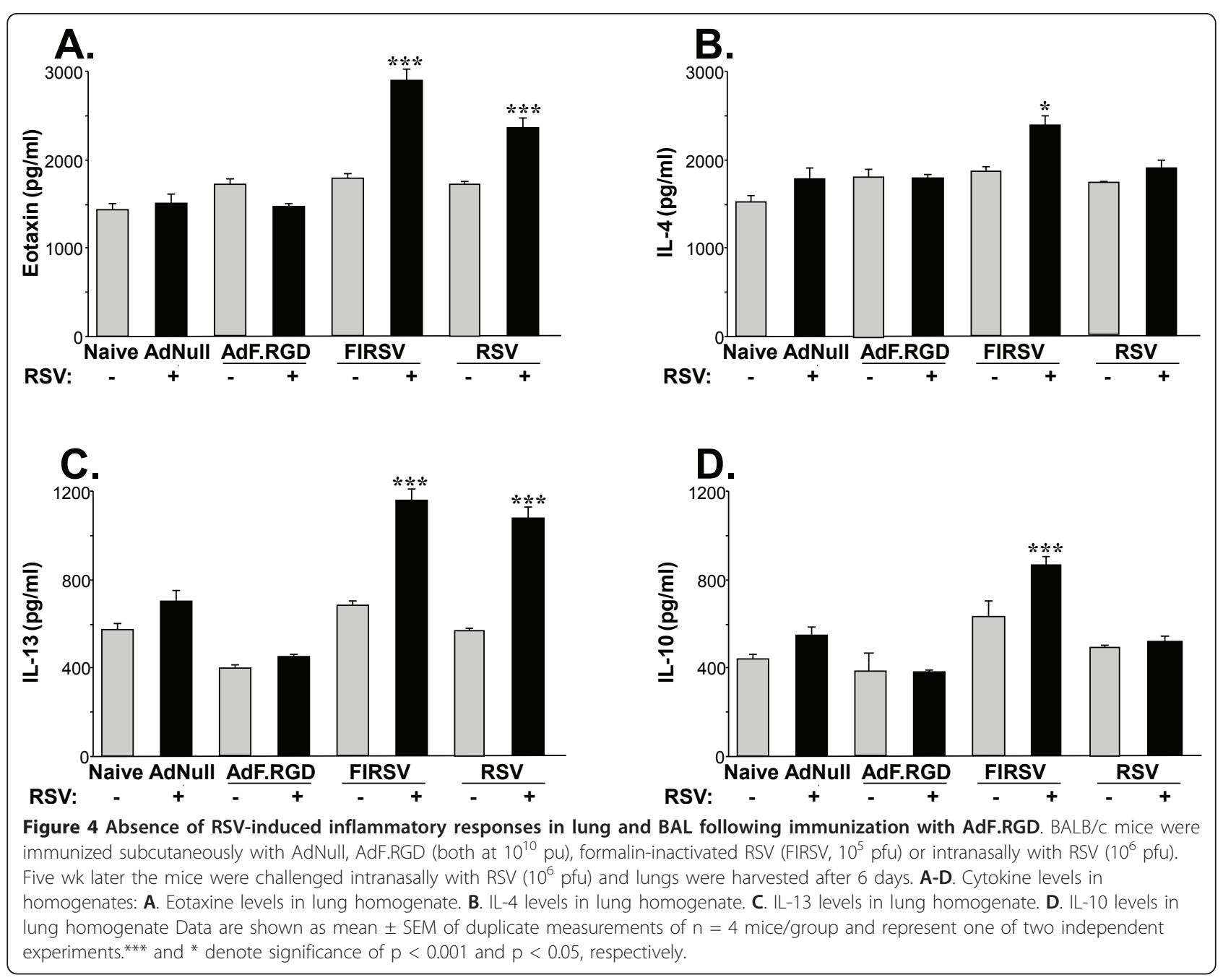

AdNull or AdF.RGD. This suggests that immunization with AdF.RGD does not trigger these features of vaccine-induced lung disease.

\section{Immunization with AdF.RGD Leads to Cellular and Protective anti-RSV Immunity}

Cellular and protective immune responses were evaluated following immunization with AdF.RGD in direct comparison to immunization with FIRSV, the vaccine that resulted in vaccine-enhanced RSV disease. BALB/c mice were immunized with AdF.RGD, AdNull or FIRSV and the systemic anti-RSV cellular immune response was evaluated 10 days after vector administration (Figure 5). The number of RSV-specific CD4 T cells secreting IL-4 from the spleen cells of mice immunized with AdF.RGD was reduced compared to mice immunized with FIRSV ( $\mathrm{p}<0.001$; Figure 5A). In contrast, AdF. RDG induced a higher RSV-specific IFN- $\gamma$ response in CD8 T cells than FIRSV (p $<0.001$; Figure 5B). Protection against RSV challenge 4 wk after immunization was similar following immunization with AdF.RGD and FIRSV ( $p>0.5$ ) compared to the AdNull control group $(\mathrm{p}<0.002$, both comparisons; Figure $5 \mathrm{C})$. These data indicate that, at the doses used, immunization with AdF. RGD and FIRSV leads to comparable levels of protective immunity, but that mice immunized with AdF.RGD have a higher Th1 and lower Th2 response than FIRSVimmunized mice and subsequently do not develop vaccine-enhanced lung disease.

\section{Characterization of Lung DC following Infection with RSV} in AdF.RGD-Immunized Mice

RSV infection results in changes in lung DC [50,56-61]. The lung $\mathrm{cDC}$ and $\mathrm{pDC}$ subsets regulate Th1 and Th2 responses, with $\mathrm{pDC}$ balancing the Th1/Th2 response via activation of Treg cells and consequently shifting the cDC-promoted Th2 response towards Th1 response [62]. To further analyze the DC composition in the lung following RSV infection, lung suspensions were prepared 6 days after intranasal administration of RSV and CDC 


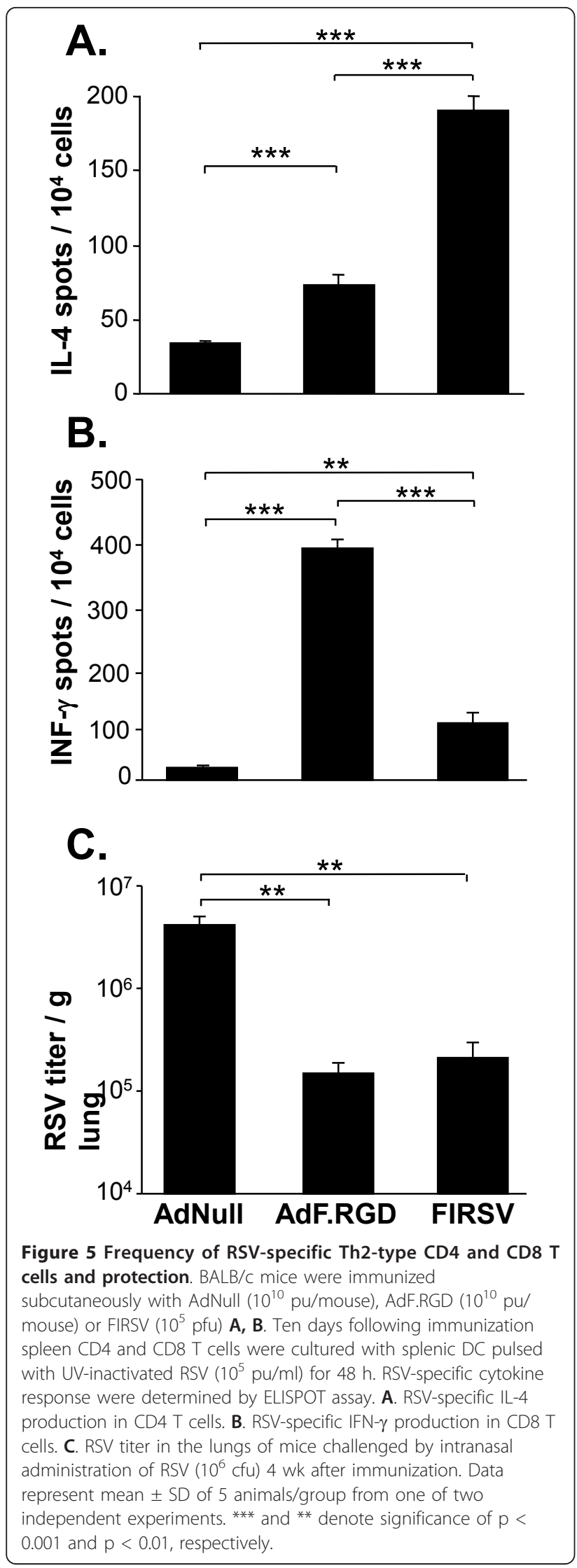

and pDC populations were analyzed by flow cytometry. Consistent with prior reports [55,57-59], the number of CD11c/CD11b-positive $\mathrm{cDC}$ and the percentage of PDCA-1-positive pDC (CD11b $\left./ C D 11 c^{+}\right)$increased after RSV infection (data not shown). To analyze the mechanism by which lung DC respond to RSV infection in AdF. RGD-immunized mice compared to mice that received the formalin-inactivated RSV vaccine, lung DC subsets were isolated 6 days following RSV infection from mice that had been immunized with AdF.RGD or FIRSV 5 wk prior to RSV infection. The $\mathrm{CDC}$ population did not significantly change between AdF.RGD and AdF.RGD plus RSV animals ( $p>0.09$; Figure $6 \mathrm{~A}$ ), indicating that vaccination with AdF.RGD does not lead to a change in the $\mathrm{CDC}$ population after RSV challenge. In contrast, cDC increased following RSV infection in the mice immunized with FIRSV compared to all the other groups ( $\mathrm{p}<0.01$, all comparisons; Figure 6A). A similar pattern was observed in the $\mathrm{pDC}$ population with the highest increase in the FIRSV-immunized mice challenged with RSV ( $p<0.01$; Figure $6 B$ ). Since viral infections are strongly controlled by IFN- $\alpha$ secretion by pDC [62], we analyzed the percentage of IFN- $\alpha$-positive pDC in the lungs of vaccinated mice following challenge with RSV. IFN- $\alpha$ positive pDC in the lungs of mice that had been immunized with AdF.RGD, AdF.RGD plus RSV and FIRSV were similar ( $\mathrm{p}>0.1$, all comparisons; Figure 6C). However, there was a significant decrease in the IFN- $\alpha$ secretion in $\mathrm{pDC}$ population in the mice immunized with FIRSV and challenged with RSV $(\mathrm{p}<0.02$; Figure $6 \mathrm{C}$ ). This indicates that $\mathrm{pDC}$ and $\mathrm{cDC}$ are increased in vaccine-induced RSV disease following immunization with FIRSV and that their imbalance could induce a Th2-promoting environment.

Since $\mathrm{pDC}$ and $\mathrm{CDC}$ function as activators and regulators for Tregs $\left(\mathrm{CD}^{+}, \mathrm{CD} 25^{\text {bright }}, \mathrm{FoxP}^{+}\right)$, a T cell population that controls the outcome of Th1 and Th2 responses $[62,63]$, we analyzed if the $C D 4^{+} C D 25$ ${ }^{+} \mathrm{FoxP}^{+}$is affected during FIRSV-induced vaccine disease and if this effect is abolished following immunization with AdF.RGD. The Treg population did not differ in the mice that had been immunized with AdF. RGD and mice that had been immunized with AdF. RGD and subsequently been challenged with RSV ( $p$ > 0.08; Figure 7A). In contrast, animals that had been immunized with FIRSV and were challenged with RSV showed a significant decrease in the Treg population $(\mathrm{p}<0.05$, all comparisons; Figure 7A). As there is a reciprocal relationship between Treg cells and IL-17 expression by Th17 cells, we evaluated the IL-17 cytokine level in the lung (Figure 7B). No significant differences were observed between mice that had been immunized with AdF.RGD and mice that had been immunized with AdF.RGD and challenged with 


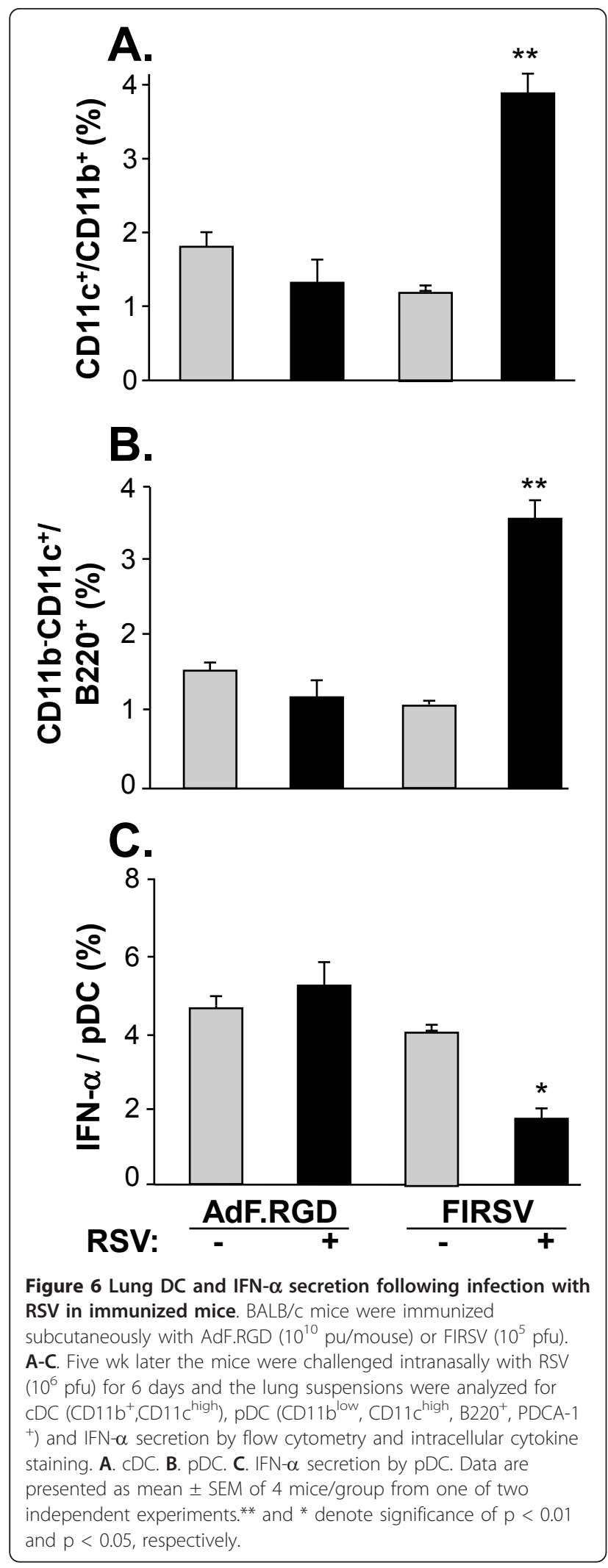

RSV ( $p>0.09$; Figure 7B). In contrast, animals that had been immunized with FIRSV and were challenged with RSV showed a significant increase in the IL-17 level in the lung ( $\mathrm{p}<0.01$, all comparisons; Figure 7B). Overall, this data suggests that the lung DC and Treg populations in vaccinated mice show distinct changes following RSV challenge, dependent upon whether the vaccine induces enhanced disease.

\section{Discussion}

Based on the exaggerated inflammatory Th2 response primed by the formalin-inactivated RSV vaccine more than 40 years ago, it has been thought that an efficient and safe vaccine against RSV should induce a Th1dominant response. Our present study shows that, compared to FIRSV, an integrin-targeted Ad vector expressing the RSV F protein induces higher levels of serum neutralizing anti-RSV antibodies with equivalent protection against RSV, but showed a decreased Th2 response and did not induce RSV disease. Immunization with FIRSV followed by RSV infection induces an imbalance in the pulmonary $\mathrm{CDC}$ and $\mathrm{pDC}$ population, diminished anti-viral activity in $\mathrm{pDC}$, and a lower frequency of regulatory $\mathrm{T}$ cells that was not present following immunization with AdF.RGD.

\section{Modification of Ad Vector to Increase Th1 Responses}

The effectiveness of Ad-based vaccines is thought to be based on the efficient infection of a variety of cells in vivo, subsequently the expression of a pathogen-specific protein encoded by the Ad expression cassette in these cells, as well as Ad vectors functioning as adjuvants $[24,25]$. Ad vectors have the capability to infect DC and this is critical for the induction of a strong anti-Ad and anti-transgene immune response [33-37]. Generating protective immunity against viruses like RSV requires the generation of humoral and cellular immunity by the vaccine. One attractive feature of Ad for genetic vaccination is the capability to modify the Ad capsid to enhance immune responses [38-40]. Targeting Ad to antigen-presenting cells by adding the RGD integrinbinding motif to the fiber knob enhances anti-transgene cellular immune responses in mice and predominantly skews cellular immune responses towards Th1, a prerequisite to develop successful anti-viral vaccines [38-40]. AdF.RGD induced neutralizing anti-RSV titer, a strong anti-RSV Th1-dominant cellular response and protected from RSV after challenge.

\section{Vaccine-induced RSV Disease}

Although many vaccine approaches, including live attenuated, viral and bacterial vectored and adjuvanted 


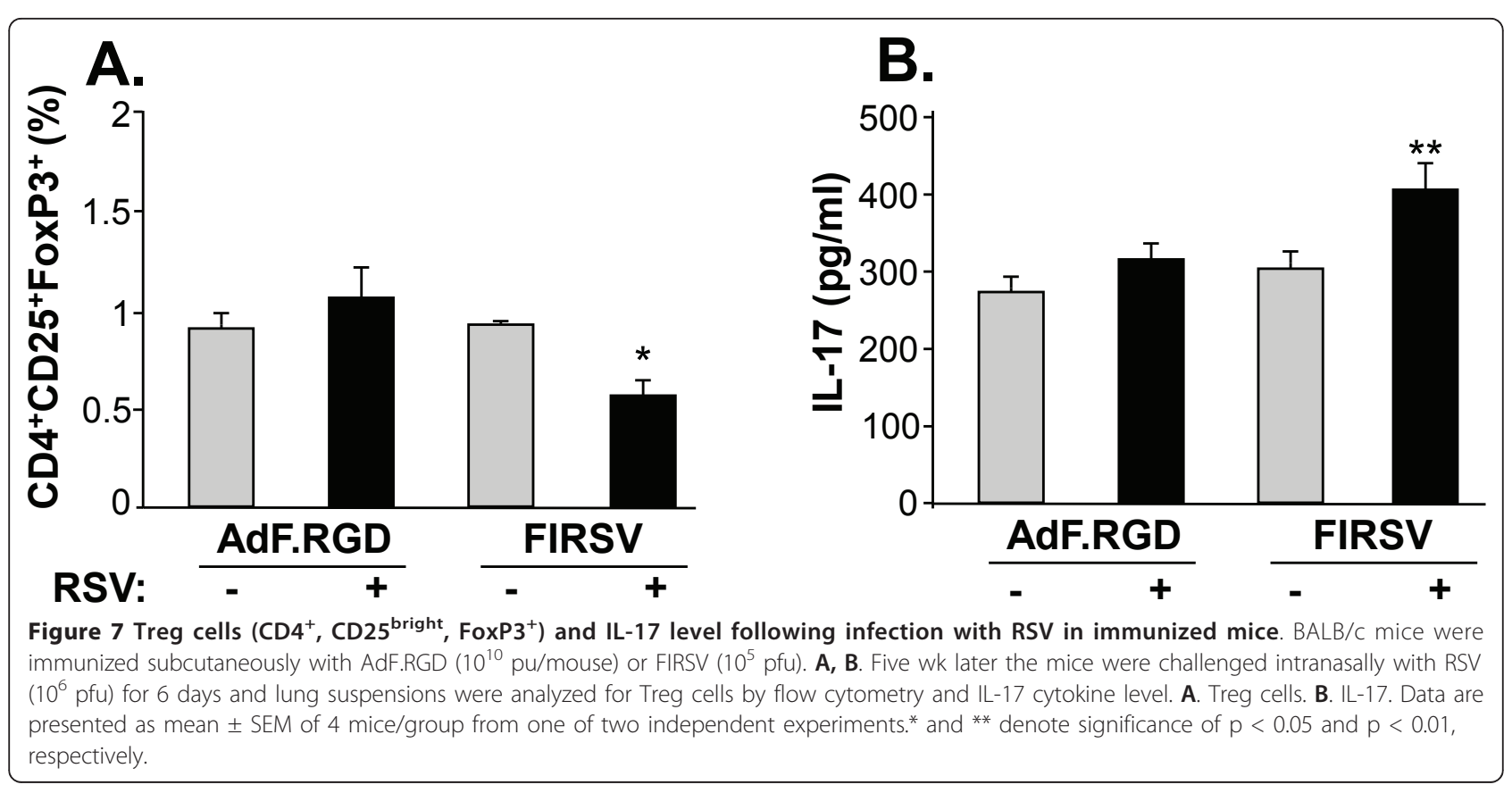

subunit vaccines, have been evaluated in rodent and primate models, there is currently no approved RSV vaccine. Screening for a live attenuated RSV vaccine candidate with the right balance of attenuation and immunogenicity has been hampered by a lack of suitable in vitro and in vivo models capable of accurately predicting attenuation in naive infants [64]. Vaccine-induced RSV disease following immunization with formalin-inactivated preparations of RSV, based on the initial clinical FIRSV vaccine, has been extensively studied in animal models responses [4-7,19,21]. Studies in cotton rats, vaccinated with FIRSV and then challenged with RSV showed infiltration with neutrophils, macrophages and lymphocytes and increased Th2-type cytokines like IL-4, IL-10, IL-13 and eotaxin in the lung, indicating a Th2 bias in enhanced inflammation $[5,65]$. The central role of $\mathrm{T}$ cells in the augmented lung pathology has been elucidated using the $\mathrm{BALB} / \mathrm{c}$ mouse model. It has been shown that $\mathrm{CD} 4 \mathrm{~T}$ cells are crucial to the immunopathogenesis of vaccine-augmented RSV disease $[4,66,67]$, and that RSV-specific antibodies (in the absence of CD4 and CD8 $\mathrm{T}$ cells) are not sufficient to cause disease enhancement $[4,66]$. It has recently been reported that immunization with FIRSV induces low avidity anti-RSV antibodies due to poor activation of toll-like receptors that could consequently result in an aberrant Th2 immune response [18]. Therefore, skewing to a Th1-type pattern of cytokine production by priming with live RSV prevented subsequent enhanced disease [67]. Studies using recombinant vaccinia viruses expressing various RSV proteins have pointed to the $G$ protein as a potential crucial factor in the induction of vaccine-enhanced disease [68]. Since the challenge lies in inducing a strong and protective $\mathrm{T}$ cell response, while avoiding the pathological consequences of unbalanced Th1/Th2 responses, Ad vectors, which tend to induce strong Th1 responses, are a promising tool for a RSV vaccine. The addition of the RGD motif to the fiber further increased the Th1 response against the $\mathrm{F}$ protein and did not induce vaccine-enhance diseased.

\section{Role of DC in Vaccine-induced RSV Disease}

It has been reported that the number of $\mathrm{cDC}$ and $\mathrm{pDC}$ is increased during RSV infection and even after apparent resolution [55,57-61]. Collectively, these data support the hypothesis that recruitment of recently attracted DCs in the lung leads to maintenance and control of the immune response in the lung, even after the actual infection and inflammation in the lung is resolved [43,50,54-56].

To date, there has been no description of the role of lung DC subsets in respiratory viral infections during the onset of vaccine-induced RSV disease. RSV-infected mice developed airway hyperreactivity as measured by a strong increase in airway resistance in response to methacholine. Depletion of pDCs around the time of RSV infection enhanced peribronchial and perivascular inflammatory infiltrates consisting of mononuclear cells and increased pro-inflammatory cytokines and decreased anti-viral secretion $[55,56]$. Mice that were immunized with FIRSV and subsequently challenged with RSV showed similar patterns of lung DC subset imbalance, resulting in down-regulation of anti-viral secretion in 
pDC as well as decreased numbers of Treg cells. Although the subset populations were increased after RSV challenge, decreased Treg cells and elevated levels of IL-17 levels indicated a Th2-type milieu which may reflect an imbalance of $\mathrm{pDC}$ and consequently $\mathrm{cDC}$ function and which was not seen following immunization with AdF.RGD. The analysis of lung DCs and Tregs may thus be useful in the evaluation for vaccineenhanced disease of new RSV vaccines.

\section{Methods}

\section{Adenovirus Vectors}

The recombinant Ad vectors used in this study are E1a-, partial E1b- and partial E3- vectors based on the Ad5 genome. AdF contains the human cytomegalovirus (CMV) intermediate early promoter/enhancer, the sequenced $F$ protein CDNA of the RSV A2 strain, same that was used for further challenge experiments, and the simian virus 40 (SV40) polyA stop signal as an expression cassette inserted into the $\mathrm{E} 1$ region. The $\mathrm{F}$ protein cDNA was kindly provided by P. Collins (Bethesda, MD). AdF.RGD has the high-affinity RGD sequence GCDCRGDCFCA incorporated at the COOH-terminal end of the fiber protein [42]. The AdNull control vector contains no transgene in the expression cassette. The vectors were used on the basis of equal number of physical particle concentration and were propagated and purified as described previously [69].

\section{Mice}

Female BALB/c mice were obtained from Taconic Farms (Tarrytown, NY). The animals were housed under specific pathogen-free conditions and used at 6 to 8 wk of age. The mice were immunized by intramuscular injection of AdF.RGD, AdNull (both at $10^{10}$ particle units; pu), FIRSV $\left(10^{5} \mathrm{pfu}\right)$, or by intranasal administration of RSV $\left(10^{5} \mathrm{pfu}\right)$. All animal studies were conducted under protocols reviewed and approved by the Weill Cornell Institutional Animal Care and Use Committee.

\section{RSV}

The RSV strain used for immunization and protection experiments was A2 (VR-1540; ATCC). FIRSV was prepared from supernatant of RSV-infected HEp-2 cells, combined with paraformaldehyde (1:1600 dilution of 37$38 \%$ stock, Sigma) to a final dilution of 1:4000, and stirred for 3 days at $37^{\circ} \mathrm{C}$. The virus was then pelleted by ultracentrifugation, resuspended, and alum-precipitated $[3,9,67]$.

\section{Cellular Immune Response}

To assess the RSV F-specific cellular immune response following immunization, BALB/c mice were immunized by intramuscular administration of either AdF, AdF.RGD, AdNull (all at $\left.10^{10} \mathrm{pu}\right)$, or FIRSV $\left(10^{5}\right.$ pfu). Unimmunized mice served as additional controls. The frequency of antigen-specific $\mathrm{T}$ lymphocytes was determined 10 days following immunization in an IFN$\gamma$ - and IL-4-specific ELISPOT assay. MAIPS-45 plates (Millipore, Bedford, MA) were coated overnight at $4^{\circ} \mathrm{C}$ with $5 \mu \mathrm{g} / \mathrm{ml}$ of cytokine-specific capture antibodies [AN18 (IFN- $\gamma$ ) or 11B11 (IL-4); Mabtech, Stockholm, Sweden]. Spleen single cell suspensions served as the source for DC, CD4 and CD8 T cells. CD4 or CD8 T cells were purified by negative depletion using SpinSep $\mathrm{T}$ cell subset purification kits (StemCell Technologies, Vancouver, BC, Canada). Purity for CD4 and CD8 T cells was between 95-98\%. Splenic DC were purified from naive animals to serve as antigen presenting cells by positive selection using CD11c MACS beads (Miltenyi Biotec, Auburn, CA) and double-purification over two consecutive MACS LS+ columns (Milentyi Biotec). The resulting DC purity was between $90-96 \%$. DC $(5 \times$ $10^{6} / \mathrm{ml}$ ) were either pulsed for $3 \mathrm{hr}$ with the $\mathrm{H}-2^{\mathrm{d}}$ restricted F epitope (F85-93, KYKNAVTEL, $100 \mu \mathrm{M}$, purity confirmed by high-performance liquid chromatography), incubated with purified recombinant RSV F protein $(100 \mu \mathrm{g} / \mathrm{ml})$, or UV-inactivated RSV $\left(10^{5} \mathrm{pu} /\right.$ $\mathrm{ml}$ ) in complete RPMI medium supplemented with 10 mM Hepes, pH 7.5 (BioSource International, Camarillo, CA) and $10^{-5} \mathrm{M}$ b-mercaptoethanol (SigmaAldrich). The recombinant RSV F protein was produced from a bacterial expression vector. A bacterial expression vector (pSmt3-RSV F) was constructed, by cloning the PCR amplified RSV F gene (forward primer: 5'-CCC CGA TCC ACA ATG GAG TTG CTA ATC CTC-3'; reverse primer: 5'-A TAA CGT AAA TCA TTG ATT TTC GAA CCC-3') into the petSUMO expression vector (Invitrogen) and the recombinant fusion protein was purified by Ni-chelating affinity chromatography according to the manufacturer's protocol (Prebound, Qiagen, Valencia, CA). Prior to addition of responder $\mathrm{T}$ cells, antibody-coated plates were blocked with complete RPMI medium supplemented with $10 \mathrm{mM}$ HEPES, pH 7.4 (BioSource International) and $10^{-5} \mathrm{M} \beta$-mercaptoethanol (SigmaAldrich) for $3 \mathrm{~h}$. CD4 $\left(2 \times 10^{5}\right)$ or CD8 $\left(10^{5}\right) \mathrm{T}$ cells were incubated with splenic DC, pulsed with either recombinant RSV F protein, F85-93 peptide, RSV, or no antigen at a $\mathrm{T}$ cell: DC ratio of $6: 1$ for $48 \mathrm{~h}$. Following washing, biotinylated anti-IFN- $\gamma$ or anti-IL-4 detection antibodies (both at $1 \mu \mathrm{g} / \mathrm{ml}$, Mabtech) were added and the plates were incubated for $2 \mathrm{~h}$ at $37^{\circ} \mathrm{C}$, followed by a streptavidin-alkaline phosphatase conjugate (Vectastain-ABC peroxidase kit, Vector Laboratories, Burlingame, CA) and a 3-amino-9-ethylcarbazole substrate (Sigma) for spot detection. The 
spots were counted by computer-assisted ELISPOT image analysis (Zellnet Consulting, New York, NY).

\section{Protection of Mice from Intranasal Challenge with RSV}

To evaluate the protection against RSV infection following immunization, $\mathrm{BALB} / \mathrm{c}$ mice were immunized by intramuscular administration of AdF.RGD, AdNull (both at $\left.10^{10} \mathrm{pu}\right)$ or FIRSV $\left(10^{5} \mathrm{pfu}\right)$, and were then challenged with RSV $\left(10^{6} \mathrm{pfu}\right)$ by intranasal inoculation after 4 wk. Four days later the mice were sacrificed, the lungs were homogenized in $1 \mathrm{ml} \mathrm{MEM}$ and the homogenates centrifuged at $1200 \mathrm{rpm}$ at $4^{\circ} \mathrm{C}$ for $10 \mathrm{~min}$. Tenfold serial dilutions of the lung homogenate supernatant were incubated in infection medium (MEM supplemented with $1 \%$ penicillin/streptomycin) on HEp2 cells for 3 $\mathrm{h}$ at $37^{\circ} \mathrm{C}$. The medium was then replaced with $1 \%$ methylcellulose in MEM containing 5\% fetal bovine serum and $1 \%$ penicillin/streptomycin). After 4 days, the cells were fixed with $4 \%$ paraformaldehyde, stained with $1 \%$ crystal violet and the plaques were counted under a microscope. The neutralization titer was calculated from the average plaques of four wells as the reciprocal of the highest dilution of serum that completely prevented RSV activity (>90\%).

\section{Neutralizing RSV Titer}

To evaluate serum anti-RSV neutralizing antibody titers, $\mathrm{BALB} / \mathrm{c}$ mice were immunized by intramuscular administration of AdF.RGD, AdNull (both at $10^{10} \mathrm{pu}$ ), FIRSV $\left(10^{5} \mathrm{pfu}\right)$, or by intranasal administration of RSV $\left(10^{5}\right.$ pfu). Sera was collected 4 wk post-administration and serial dilutions of the sera in infection medium were incubated with RSV (strain A2) for $1 \mathrm{~h}$ at $37^{\circ} \mathrm{C}$. They were then incubated on HEp2 cells for $90 \mathrm{~min}$ at $37^{\circ} \mathrm{C}$. The medium was then changed to $1 \%$ methylcellulose medium and incubated for 4 days. The cells were fixed and the titers quantified as outlined above.

\section{Vaccine-enhanced RSV Disease}

To evaluate vaccine-enhanced RSV pulmonary disease, mice were immunized by intramuscular administration of AdF.RGD, AdNull (both at $\left.10^{10} \mathrm{pu}\right)$, FIRSV $\left(10^{5} \mathrm{pfu}\right)$, or by intranasal administration of RSV $\left(10^{5} \mathrm{pfu}\right)$. The mice were challenged with RSV at 5 wk post-immunization and sacrificed 6 days later. Lungs were fixed in $4 \%$ paraformaldehyde at a constant pressure of $25 \mathrm{~cm} \mathrm{H}_{2} \mathrm{O}$ for $4 \mathrm{hr}$. Histological sections were stained with hematoxylin and eosin and evaluated in a blinded study for inflammatory changes by light microscopy. A second experimental group of mice was sacrificed at 4 days following RSV infection for evaluation of cell content and IL-4, IL-10, IL-13, IL-17 and eotaxin concentrations by ELISA (R\&D System, MN).

\section{Lung DC and Tregs}

$\mathrm{BALB} / \mathrm{c}$ mice were immunized with AdF.RGD, AdNull (all at $\left.10^{10} \mathrm{pu}\right)$, FIRSV $\left(10^{5} \mathrm{pfu}\right)$ or RSV $\left(10^{5} \mathrm{pfu}\right) 5 \mathrm{wk}$ prior to intranasal challenge with RSV $\left(10^{5} \mathrm{pu}\right)$. Lungs were harvested 6 days post-challenge, digested for $20 \mathrm{~min}$ at $37^{\circ} \mathrm{C}$ with DNAseI and Collagenase (Sigma-Aldrich, $\mathrm{MO}$ ), and passed through a cell strainer. Myeloid and lymphoid DC were characterized as CD11b $\mathrm{b}^{+}, \mathrm{CD} 11 \mathrm{c}^{\text {bright }}$ cells (conventional DC, $\mathrm{CDC}$ ), and $\mathrm{pDC}$ were characterized as $\mathrm{CD} 11 \mathrm{~b}^{\text {low }}, \mathrm{CD} 11 \mathrm{c}^{\text {bright }}, \mathrm{B} 220^{+}$and confirmed as PDCA $-1^{+}$cells by flow cytometry (BD Bioscience, CA). IFN- $\alpha$ secretion by $\mathrm{pDC}$ was determined by intracellular cytokine staining. Briefly, cells were fixed and permeabilized with Cytofix/Cytoperm reagent (BD Biosciences) for $20 \mathrm{~min}$ at $4^{\circ} \mathrm{C}$, then washed twice in Perm/Wash solution (BD Biosciences). The cells were then stained (30 min, $4^{\circ}$ C) for intracellular cytokine using phycoerythrin (PE)conjugated monoclonal antibody against murine cytokine IFN- $\alpha$. CD $4^{+}, \mathrm{CD} 25^{\text {bright }}$, FoxP3 $^{+}$Tregs from the lung were analyzed 6 days post-challenge with RSV by costaining the lung suspensions with allophyocyanine-conjugated anti-murine $\mathrm{CD} 4$, phycoerythrin-conjugated antimurine CD25 and fluorescein-conjugated anti-murine FoxP3 antibodies (BD Bioscience). Cells were then analyzed by flow cytometry using a FACSCalibur flow cytometer (Becton Dickinson, NJ).

\section{Statistics}

The data are presented as mean \pm standard error of the mean. Statistical analyses were performed using a nonpaired two-tailed Student's t-test. Statistical significance was determined at $\mathrm{p}<0.05$.

\section{Note}

₹ These studies were supported, in part, by R01 A1059228.

\section{Acknowledgements \\ We thank Peter Collins for providing the RSV plasmid, Kyung Hee Kim for technical assistance and Bishnu De for helpful suggestions.}

\section{Author details}

${ }^{1}$ Department of Genetic Medicine, Weill Medical College of Cornell University, New York, New York, USA. ²Department of Pediatrics, Weill Medical College of Cornell University, New York, New York, USA.

\section{Authors' contributions}

AK designed this study, participated in the experiments and drafted and edited the manuscript, YX carried out the experiments and participated in discussions, SR participated in the experiments, WW carried out the experiments, JJ participated in the experiments, SW designed this study and edited the manuscript. All authors read and approved the final manuscript.

\section{Competing interests}

The authors declare that they have no competing interests.

Received: 20 May 2011 Accepted: 29 July 2011 Published: 29 July 2011 


\section{References}

1. Collins PL, Crowe JE Jr: Respiratory syncytial virus and metapneumovirus. In Fields Virology. Edited by: Knipe DM, Howley P. Philadelphia: LippincottWilliams and Wilkins, Wolters Kluwer Business; 2007:1601-1646.

2. Kapikian AZ, Mitchell RH, Chanock RM, Shvedoff RA, Stewart CE: An epidemiologic study of altered clinical reactivity to respiratory syncytial (RS) virus infection in children previously vaccinated with an inactivated RS virus vaccine. Am J Epidemiol 1969, 89:405-421.

3. Kim HW, Canchola JG, Brandt CD, Pyles G, Chanock RM, Jensen K, et al: Respiratory syncytial virus disease in infants despite prior administration of antigenic inactivated vaccine. Am J Epidemiol 1969, 89:422-434.

4. Castilow EM, Olson MR, Varga SM: Understanding respiratory syncytial virus (RSV) vaccine-enhanced disease. Immunol Res 2007, 39:225-239.

5. Openshaw PJ, Tregoning JS: Immune responses and disease enhancement during respiratory syncytial virus infection. Clin Microbiol Rev 2005, 18:541-555.

6. Polack FP, Teng MN, Collins PL, Prince GA, Exner $M$, Regele $H$, et al: A role for immune complexes in enhanced respiratory syncytial virus disease. $J$ Exp Med 2002, 196:859-865

7. van Drunen Littel-van den Hurk, Mapletoft JW, Arsic N, Kovacs-Nolan J: Immunopathology of RSV infection: prospects for developing vaccines without this complication. Rev Med Virol 2007, 17:5-34

8. Connors M, Giese NA, Kulkarni AB, Firestone CY, Morse HC III, Murphy BR: Enhanced pulmonary histopathology induced by respiratory syncytial virus (RSV) challenge of formalin-inactivated RSV-immunized BALB/C mice is abrogated by depletion of interleukin-4 (IL-4) and IL-10. J Virol 1994, 68:5321-5325.

9. Graham BS, Henderson GS, Tang YW, Lu X, Neuzil KM, Colley DG: Priming immunization determines $T$ helper cytokine mRNA expression patterns in lungs of mice challenged with respiratory syncytial virus. J Immunol 1993, 151:2032-2040

10. Power UF, Huss T, Michaud V, Plotnicky-Gilquin H, Bonnefoy JY, Nguyen TN: Differential histopathology and chemokine gene expression in lung tissues following respiratory syncytial virus (RSV) challenge of formalininactivated RSV- or BBG2Na-immunized mice. J Virol 2001, 75:12421-12430.

11. Hussell T, Baldwin CJ, O'Garra A, Openshaw PJ: CD8+ T cells control Th2driven pathology during pulmonary respiratory syncytial virus infection. Eur J Immunol 1997, 27:3341-3349.

12. Hussell T, Openshaw PJ: Intracellular IFN-gamma expression in natural killer cells precedes lung CD8+ T cell recruitment during respiratory syncytial virus infection. J Gen Virol 1998, 79(Pt 11):2593-2601.

13. Srikiatkhachorn A, Braciale TJ: Virus-specific CD8+ T lymphocytes downregulate $T$ helper cell type 2 cytokine secretion and pulmonary eosinophilia during experimental murine respiratory syncytial virus infection. J Exp Med 1997, 186:421-432.

14. Olson MR, Varga SM: CD8 T Cells Inhibit Respiratory Syncytial Virus (RSV) Vaccine-Enhanced Disease. The Journal of Immunology 2007, 179:5415-5424.

15. Johnson TR, Graham BS: Contribution of respiratory syncytial virus $G$ antigenicity to vaccine-enhanced illness and the implications for severe disease during primary respiratory syncytial virus infection. Pediatr Infect Dis J 2004, 23:S46-S57.

16. Johnson TR, Graham BS: Secreted Respiratory Syncytial Virus G Glycoprotein Induces Interleukin-5 (IL-5), IL-13, and Eosinophilia by an IL-4-Independent Mechanism. J Virol 1999, 73:8485-8495.

17. Johnson TR, Rothenberg ME, Graham BS: Pulmonary eosinophilia requires interleukin-5, eotaxin-1, and CD4+ T cells in mice immunized with respiratory syncytial virus $\mathrm{G}$ glycoprotein. Journal of Leukocyte Biology 2008, jlb..

18. Delgado MF, Coviello S, Monsalvo AC, Melendi GA, Hernandez JZ, Batalle JP, et al: Lack of antibody affinity maturation due to poor Toll-like receptor stimulation leads to enhanced respiratory syncytial virus disease. Nat Med 2009, 15:34-41.

19. Castilow EM, Legge $K L$, Varga SM: Cutting edge: Eosinophils do not contribute to respiratory syncytial virus vaccine-enhanced disease. J Immunol 2008, 181:6692-6696.

20. Greenberg HB, Piedra PA: Immunization against viral respiratory disease: a review. Pediatr Infect Dis J 2004, 23:S254-S261.

21. Piedra PA: Clinical experience with respiratory syncytial virus vaccines Pediatr Infect Dis J 2003, 22:S94-S99.
22. Kneyber MC, Kimpen $J$ : Advances in respiratory syncytial virus vaccine development. Curr Opin Investig Drugs 2004, 5:163-170.

23. Singh SR, Dennis VA, Carter CL, Pillai SR, Moore EG: Respiratory Syncytial Virus Recombinant F Protein (Residues 255-278) Induces a Helper T Cell Type 1 Immune Response in Mice. Viral Immunology 2007, 20:261-275.

24. Hackett NR, Kaminsky SM, Sondhi D, Crystal RG: Antivector and antitransgene host responses in gene therapy. Curr Opin Mol Ther 2000, 2:376-382

25. Zhou D, Ertl HC: Therapeutic potential of adenovirus as a vaccine vector for chronic virus infections. Expert Opin Biol Ther 2006, 6:63-72.

26. Collins PL, Davis AR, Lubeck MD, Mizutani S, Hung PP, Prince GA, et al: Evaluation of the protective efficacy of recombinant vaccinia viruses and adenoviruses that express respiratory syncytial virus glycoproteins. In Vaccines 90: Modern approaches to new vaccines including prevention of AIDS. Edited by: Brown F. Cold Spring Harbor, NY: Cold Spring Harbor Laboratory Press; 1990:79.

27. Fu Y, He J, Zheng X, Wu Q, Zhang M, Wang X, et al: Intranasal immunization with a replication-deficient adenoviral vector expressing the fusion glycoprotein of respiratory syncytial virus elicits protective immunity in BALB/c mice. Biochem Biophys Res Commun 2009, 381:528-532.

28. Hsu KH, Lubeck MD, Davis AR, Bhat RA, Selling BH, Bhat BM, et al: Immunogenicity and protective efficacy of adenovirus vectored respiratory syncytial virus vaccine. In Vaccines 91: Modern approaches to new vaccines including prevention of AIDS. Edited by: Chanock RM, Ginsberg HS, Brown F, Lerner RA. Cold Spring Harbor, NY: Cold Spring Harbor Laboratory; 1991:293-297.

29. Hsu KH, Lubeck MD, Davis AR, Bhat RA, Selling BH, Bhat BM, et al: Immunogenicity of recombinant adenovirus-respiratory syncytial virus vaccines with adenovirus types 4, 5, and 7 vectors in dogs and a chimpanzee. J Infect Dis 1992, 166:769-775.

30. Hsu KH, Lubeck MD, Bhat BM, Bhat RA, Kostek B, Selling BH, et al: Efficacy of adenovirus-vectored respiratory syncytial virus vaccines in a new ferret model. Vaccine 1994, 12:607-612.

31. Shao HY, Yu SL, Sia C, Chen Y, Chitra E, Chen $H$, et al: Immunogenic properties of RSV-B1 fusion (F) protein gene-encoding recombinant adenoviruses. Vaccine 2009, 27:5460-5471.

32. Yu JR, Kim S, Lee JB, Chang J: Single Intranasal Immunization with Recombinant Adenovirus-Based Vaccine Induces Protective Immunity against Respiratory Syncytial Virus Infection. The Journal of Virology 2008 82:2350-2357.

33. Dietz $A B$, Vuk-Pavlovic S: High efficiency adenovirus-mediated gene transfer to human dendritic cells. Blood 1998, 91:392-398.

34. Herrera OB, Brett S, Lechler RI: Infection of mouse bone marrow-derived dendritic cells with recombinant adenovirus vectors leads to presentation of encoded antigen by both MHC class I and class II molecules-potential benefits in vaccine design. Vaccine 2002 , 21:231-242.

35. Jooss K, Yang Y, Fisher KJ, Wilson JM: Transduction of dendritic cells by DNA viral vectors directs the immune response to transgene products in muscle fibers. J Virol 1998, 72:4212-4223.

36. Labow D, Lee S, Ginsberg RJ, Crystal RG, Korst RJ: Adenovirus vectormediated gene transfer to regional lymph nodes. Hum Gene Ther 2000, 11:759-769.

37. Mercier S, Gahery-Segard H, Monteil M, Lengagne R, Guillet JG, Eloit M, et al: Distinct Roles of Adenovirus Vector-Transduced Dendritic Cells, Myoblasts, and Endothelial Cells in Mediating an Immune Response against a Transgene Product. The Journal of Virology 2002, 76:2899-2911.

38. Harui A, Roth MD, Vira D, Sanghvi M, Mizuguchi H, Basak SK: Adenoviralencoded antigens are presented efficiently by a subset of dendritic cells expressing high levels of alpha(v)beta3 integrins. J Leukoc Biol 2006, 79:1271-1278.

39. Okada N, Saito T, Masunaga Y, Tsukada Y, Nakagawa S, Mizuguchi H, et al: Efficient antigen gene transduction using Arg-Gly-Asp fiber-mutant adenovirus vectors can potentiate antitumor vaccine efficacy and maturation of murine dendritic cells. Cancer Res 2001, 61:7913-7919.

40. Worgall S, Busch A, Rivara M, Bonnyay D, Leopold PL, Merritt R, et al: Modification to the capsid of the adenovirus vector that enhances dendritic cell infection and transgene-specific cellular immune responses. J Virol 2004, 78:2572-2580 
41. Roelvink PW, Mi Lee G, Einfeld DA, Kovesdi I, Wickham TJ: Identification of a conserved receptor-binding site on the fiber proteins of CARrecognizing adenoviridae. Science 1999, 286:1568-1571.

42. Wickham TJ, Tzeng E, Shears LL2, Roelvink PW, Li Y, Lee GM, et al: Increased in vitro and in vivo gene transfer by adenovirus vectors containing chimeric fiber proteins. J Virol 1997, 71:8221-8229.

43. de Heer HJ, Hammad H, Kool M, Lambrecht BN: Dendritic cell subsets and immune regulation in the lung. Semin Immunol 2005, 17:295-303.

44. GeurtsvanKessel CH, Lambrecht BN: Division of labor between dendritic cell subsets of the lung. Mucosal Immunol 2008, 1:442-450.

45. Segura E, Villadangos JA: Antigen presentation by dendritic cells in vivo. Curr Opin Immunol 2009, 21:105-110.

46. von Garnier C, Nicod LP: Immunology taught by lung dendritic cells. Swiss Med Wkly 2009, 139:186-192.

47. Webb TJ: The phenotype and function of lung dendritic cells. Critical reviews in immunology 2005, 25:465-491.

48. Kelsall BL, Biron CA, Sharma O, Kaye PM: Dendritic cells at the hostpathogen interface. Nat Immunol 2002, 3:699-702.

49. Shortman K, Liu YJ: Mouse and human dendritic cell subtypes. Nat Rev Immunol 2002, 2:151-161.

50. Smit JJ, Lindell DM, Boon L, Kool M, Lambrecht BN, Lukacs NW: The Balance between Plasmacytoid DC versus Conventional DC Determines Pulmonary Immunity to Virus Infections. PLOS ONE 2008, 3:e1720.

51. Webb TJ, Sumpter TL, Thiele AT, Swanson KA, Wilkes DS: The phenotype and function of lung dendritic cells. Crit Rev Immunol 2005, 25:465-491.

52. Wu L, Liu YJ: Development of dendritic-cell lineages. Immunity 2007, 26:741-750.

53. Lambrecht BN, De VM, Coyle AJ, Gutierrez-Ramos JC, Thielemans K, Pauwels RA: Myeloid dendritic cells induce Th2 responses to inhaled antigen, leading to eosinophilic airway inflammation. J Clin Invest 2000, 106:551-559.

54. Schlender J, Hornung V, Finke S, Gunthner-Biller M, Marozin S, Brzozka K, et al: Inhibition of Toll-Like Receptor 7- and 9-Mediated Alpha/Beta Interferon Production in Human Plasmacytoid Dendritic Cells by Respiratory Syncytial Virus and Measles Virus. The Journal of Virology 2005, 79:5507-5515.

55. Smit JJ, Rudd BD, Lukacs NW: Plasmacytoid dendritic cells inhibit pulmonary immunopathology and promote clearance of respiratory syncytial virus. J Exp Med 2006, 203:1153-1159.

56. Wang $H$, Peters $N$, Schwarze J: Plasmacytoid dendritic cells limit viral replication, pulmonary inflammation, and airway hyperresponsiveness in respiratory syncytial virus infection. J Immunol 2006, 177:6263-6270.

57. Beyer M, Bartz H, Horner K, Doths S, Koerner-Rettberg C, Schwarze J: Sustained increases in numbers of pulmonary dendritic cells after respiratory syncytial virus infection. J Allergy Clin Immunol 2004, 113:127-133.

58. Guerrero-Plata A, Kolli D, Hong C, Casola A, Garofalo RP: Subversion of pulmonary dendritic cell function by paramyxovirus infections. J Immunol 2009, 182:3072-3083.

59. Lukens MV, Kruijsen D, Coenjaerts FE, Kimpen $J$, van Bleek GM: Respiratory syncytial virus-induced activation and migration of respiratory dendritic cells and subsequent antigen presentation in the lung-draining lymph node. J Virol 2009, 83:7235-7243.

60. Gill MA, Palucka AK, Barton T, Ghaffar F, Jafri H, Banchereau J, et al: Mobilization of plasmacytoid and myeloid dendritic cells to mucosal sites in children with respiratory syncytial virus and other viral respiratory infections. J Infect Dis 2005, 191:1105-1115.

61. Boogaard I, van Oosten M, van Rijt LS, Muskens F, Kimman TG, Lambrecht BN, et al: Respiratory syncytial virus differentially activates murine myeloid and plasmacytoid dendritic cells. Immunology 2007, 122:65-72.

62. Ouabed A, Hubert FX, Chabannes D, Gautreau L, Heslan M, Josien R: Differential control of $\mathrm{T}$ regulatory cell proliferation and suppressive activity by mature plasmacytoid versus conventional spleen dendritic cells. J Immunol 2008, 180:5862-5870.

63. Ruckwardt TJ, Bonaparte KL, Nason MC, Graham BS: Regulatory T Cells Promote Early Influx of CD8+ T Cells in the Lungs of Respiratory Syncytial Virus-Infected Mice and Diminish Immunodominance Disparities. The Journal of Virology 2009, 83:3019-3028.

64. Wright PF, Karron RA, Belshe RB, Shi JR, Randolph VB, Collins PL, et al: The absence of enhanced disease with wild type respiratory syncytial virus infection occurring after receipt of live, attenuated, respiratory syncytial virus vaccines. Vaccine 2007, 25:7372-7378.

65. Connors M, Collins PL, Firestone CY, Sotnikov AV, Waitze A, Davis AR, et al: Cotton rats previously immunized with a chimeric RSV FG glycoprotein develop enhanced pulmonary pathology when infected with RSV, a phenomenon not encountered following immunization with vacciniaRSV recombinants or RSV. Vaccine 1992, 10:475-484.

66. Connors M, Kulkarni AB, Firestone CY, Holmes KL, Morse HC III, Sotnikov AV, et al: Pulmonary histopathology induced by respiratory syncytial virus (RSV) challenge of formalin-inactivated RSV-immunized BALB/c mice is abrogated by depletion of CD4+ T cells. J Virol 1992, 66:7444-7451.

67. Waris ME, Tsou C, Erdman DD, Zaki SR, Anderson LJ: Respiratory synctial virus infection in BALB/c mice previously immunized with formalininactivated virus induces enhanced pulmonary inflammatory response with a predominant Th2-like cytokine pattern. J Virol 1996, 70:2852-2860.

68. Wyatt LS, Whitehead SS, Venanzi KA, Murphy BR, Moss B: Priming and boosting immunity to respiratory syncytial virus by recombinant replication-defective vaccinia virus MVA. Vaccine 1999, 18:392-397.

69. Hersh J, Crystal RG, Bewig B: Modulation of gene expression after replication-deficient, recombinant adenovirus-mediated gene transfer by the product of a second adenovirus vector. Gene Ther 1995, 2:124-131.

doi:10.1186/1743-422X-8-375

Cite this article as: Krause et al:: Absence of vaccine-enhanced RSV disease and changes in pulmonary dendritic cells with adenovirusbased RSV vaccine. Virology Journal 2011 8:375.

\section{Submit your next manuscript to BioMed Central and take full advantage of:}

- Convenient online submission

- Thorough peer review

- No space constraints or color figure charges

- Immediate publication on acceptance

- Inclusion in PubMed, CAS, Scopus and Google Scholar

- Research which is freely available for redistribution

Submit your manuscript at www.biomedcentral.com/submit
C) Biomed Central 